\title{
Solvent controlled charge transfer dynamics on diabatic surfaces with different curvatures
}

\author{
J. Casado-Pascual ${ }^{\text {a,b }}$, I. Goychuk ${ }^{\text {b }}$, M. Morillo ${ }^{\text {a }}$, P. Hänggi b,* \\ ${ }^{a}$ Física Teórica, Universidad de Sevilla, Apartado de Correos 1065, Sevilla 41080, Spain \\ ${ }^{\mathrm{b}}$ Institut für Physik, Universität Augsburg, Universitätsstraße 1, D-86135 Augsburg, Germany
}

Received 6 March 2002; in final form 17 May 2002

\begin{abstract}
We investigate electron transfer reactions on diabatic surfaces with different curvatures within the framework of Zusman theory. First, a generalization of the nonadiabatic Marcus-Levich-Dogonadze rate expression is obtained for the case of different forward and backward reorganization energies. Second, we provide a corresponding generalization of the Zusman rate expression which bridges between nonadiabatic and solvent controlled adiabatic electron transfer. The derived analytical rate expressions compare favorably against the precise numerics. A detailed comparison with previous work of Tang is presented. (c) 2002 Elsevier Science B.V. All rights reserved.
\end{abstract}

The kinetics of electron transfer (ET) processes in condensed media is essentially mediated by solvent effects. In the theories developed by Marcus [1-3], Hush [4], Levich and Dogonadze [5] the possible influence of the solvent dynamics on the transfer rate is neglected. Over 20 years ago, Zusman [6] and Alexandrov [7] introduced a set of equations to incorporate the important solvent relaxation effects. In the Zusman-Alexandrov theory, the limits of nonadiabatic and solvent controlled adiabatic transfer are described in a unified way. In the theoretical analysis, the reaction coordinate is usually assumed to move on parabolic diabatic energy curves of identical curvatures. The curva-

\footnotetext{
${ }^{*}$ Corresponding author. Fax: +49-821-598-3222.

E-mail addresses: peter.hanggi@physik.uni-augsburg.de, hanggi@physik.uni-augsburg.de (P. Hänggi).
}

ture is related to the reorganization energy, so that equal curvatures implies that the reorganization energies for the forward and backward reactions are identical. Previous analytical studies and numerical simulations indicate that the values of these two reorganization energies can be different [8-13]. A few years ago, Tang [14] presented an extension of the Zusman-Alexandrov theory to the case of parabolic diabatic energy curves of different curvatures. Tang's analysis relies on the use of the contact approximation (cf. Eq. (11)). The main objective of our study is to further extend the analysis of Zusman equations to the case of two parabolas with different curvatures beyond the contact approximation and to compare the analytical predictions with the numerical solutions of Zusman equations. In the limit of nonadiabatic electron transfer we present a rate expression which is favorably tested against numerical solutions of 
Zusman equations. We will also obtain a generalization of the Zusman rate expression that allows us to span the nonadiabatic to solvent controlled adiabatic electron transfer regimes in a unified way. Our expression is compared with a result obtained by Tang [14].

The basic elements to describe electron transfer processes are two diabatic electronic energy curves $V_{j}(x), j=1,2$, and a generalized one-dimensional reaction coordinate $x$ with effective mass $m$. The electronic states before and after the charge transfer will be denoted as donor, $|1\rangle$, and acceptor, $|2\rangle$, respectively. The reaction coordinate represents a combination of the selected nuclear modes coupled directly to the electronic transfer system [15]. The reaction coordinate is also coupled to the rest of nuclear modes. This coupling introduces friction in the dynamics of the reaction coordinate. In the overdamped limit, Zusman equations provide an appropriate description for the time evolution of the matrix elements $\rho_{j k}(x, t):=\langle j, x|\hat{\rho}(t)| x, k\rangle$ of the reduced density operator in the electron and reaction coordinate Hilbert space. Zusman equations and their validity conditions have been repeatedly derived and discussed in the literature [16-21]. These equations read in matrix form

$$
\begin{aligned}
\frac{\partial}{\partial t} \boldsymbol{\rho}(x, t)= & -\frac{\mathrm{i}}{2 \hbar}\left[\left(V_{1}(x)-V_{2}(x)\right) \boldsymbol{\sigma}_{z}+\Delta \boldsymbol{\sigma}_{x}, \boldsymbol{\rho}(x, t)\right]_{-} \\
& +D \frac{\partial}{\partial x}\left\{\frac{\partial}{\partial x}+\frac{V_{1}^{\prime}(x)+V_{2}^{\prime}(x)}{2 k_{\mathrm{B}} T}\right\} \boldsymbol{\rho}(x, t) \\
& +\frac{D}{4 k_{\mathrm{B}} T} \frac{\partial}{\partial x}\left\{\left(V_{1}^{\prime}(x)-V_{2}^{\prime}(x)\right)\left[\boldsymbol{\sigma}_{z}, \boldsymbol{\rho}(x, t)\right]_{+}\right\},
\end{aligned}
$$

where $[,]_{+}$and $[,]_{-}$represent, respectively, the anticommutator and the commutator, $\boldsymbol{\sigma}_{\boldsymbol{x}}$ and $\boldsymbol{\sigma}_{z}$ denote the standard Pauli matrices, $D$ is the diffusion coefficient in the Smoluchowski equation describing relaxation within each well, $\Delta$ denotes the electronic coupling matrix element and $k_{\mathrm{B}} T$ is the thermal energy. We will also assume parabolic diabatic curves of the form $V_{j}(x)=m \omega_{j}^{2}(x-$ $\left.x_{0} \delta_{j, 2}\right)^{2} / 2-\epsilon_{0} \delta_{j, 2}(j=1,2)$, where $x_{0}$ and $\epsilon_{0}$ are the horizontal and vertical shifts, respectively, between the minima of the parabolas (see Fig. 1), and $\omega_{j}^{2}$ characterizes the curvature of each well. In the

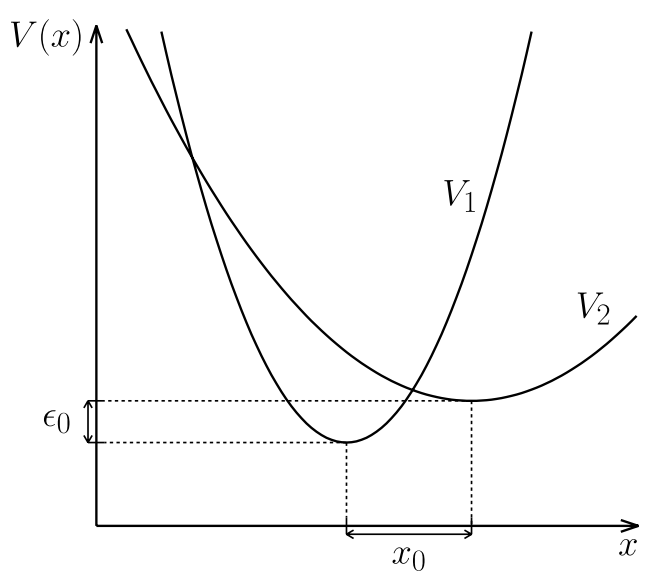

Fig. 1. Sketch of the two diabatic energy surfaces $V_{1}(x)$ and $V_{2}(x)$ possessing different curvatures. The number of crossing points can be controlled by varying the energy bias $\epsilon_{0}$.

energetics of ET reactions the reorganization energies $\lambda_{j}=m \omega_{j}^{2} x_{0}^{2} / 2$ play a fundamental role. Electron tunneling is most effective near the crossing points, cf. Fig. 1, of the curves, which are given by

$x_{j}^{*}=\frac{x_{0}}{\lambda_{2}-\lambda_{1}}\left[\lambda_{2}+(-1)^{j} \sqrt{\left(1-\frac{\epsilon_{0}}{\epsilon_{\mathrm{c}}}\right) \lambda_{1} \lambda_{2}}\right]$,

with $\epsilon_{\mathrm{c}}=\lambda_{1} \lambda_{2} /\left(\lambda_{1}-\lambda_{2}\right)$. Depending on the relative values of $\epsilon_{0}$ and $\epsilon_{\mathrm{c}}$ there can be either two, one or no crossing points. Moreover, in Eq. (1) it is assumed that the linear friction force experienced by the reaction coordinate is identical in both diabatic states. The corresponding friction coefficient, $\eta$, is connected with the diffusion constant, $D$, by the Einstein relation $D=k_{\mathrm{B}} T / \eta$. Then, the overdamped harmonic oscillators corresponding to the diabatic curves $V_{1,2}(x)$ have different relaxation times $\tau_{1,2}=k_{\mathrm{B}} T /\left(m \omega_{1,2}^{2} D\right)$. Note that these relaxation times are related to the reorganization energies by $\tau_{1} / \tau_{2}=\lambda_{2} / \lambda_{1}$. Thus, to study the effect of friction on the electron transfer rate it is convenient to combine the two relaxation times into a single relaxation time $\tau$ defined through $2 / \tau:=$ $1 / \tau_{1}+1 / \tau_{2}$. Note that $\tau \propto \eta$ and $\tau_{1}=\frac{1}{2}\left(1+\lambda_{2} /\right.$ $\left.\lambda_{1}\right) \tau, \tau_{2}=\frac{1}{2}\left(1+\lambda_{1} / \lambda_{2}\right) \tau$.

In kinetics, the time evolution of the well populations, $p_{j j}(t)=\int_{-\infty}^{\infty} \mathrm{d} x \rho_{j j}(x, t)$, is given by

$p_{j j}(t)=p_{j j}(\infty)+\left[p_{j j}(0)-p_{j j}(\infty)\right] \exp (-\Gamma t)$, 
where $\Gamma$ is the rate constant and $p_{j j}(\infty)$ denotes the $j$ th well equilibrium population. In the following, we are interested in obtaining analytical expressions for these quantities. The procedure follows a generalization of techniques previously presented in the literature [17,19]; being technically rather cumbersome it will be detailed elsewhere [22]. Basically, the whole procedure goes as follows. Solving formally the off-diagonal equations using the corresponding Green functions and substituting the formal solution into the diagonal equations yields a closed set of integro-differential equations. Assuming further that diagonal and off-diagonal terms possess different, clear-cut time and space scales, one obtains a set of two differential equations for the diagonal terms that are local in space and time. These equations read $[17,19]$ :

$$
\begin{aligned}
\frac{\partial}{\partial t} \rho_{11}(x, t)= & -K(x)\left[\rho_{11}(x, t)-\rho_{22}(x, t)\right] \\
& +\mathbf{L}_{1} \rho_{11}(x, t), \\
\frac{\partial}{\partial t} \rho_{22}(x, t)= & K(x)\left[\rho_{11}(x, t)-\rho_{22}(x, t)\right] \\
& +\mathbf{L}_{2} \rho_{22}(x, t),
\end{aligned}
$$

where $\mathbf{L}_{1,2}$ are the Smoluchowski operators

$$
\mathbf{L}_{1,2}=D \frac{\partial}{\partial x}\left(\frac{\partial}{\partial x}+\frac{V_{1,2}^{\prime}(x)}{k_{\mathrm{B}} T}\right)
$$

and $K(x)$ is expressed in terms of the off-diagonal Green function. For high enough temperature, this function is only appreciably different from zero around the crossing points.

By analogy with the case of equal curvatures, the total ET dynamics can be divided into two main steps $[6,16,17,19]$. First, the stochastic dynamics of the reaction coordinate brings the donor and acceptor states near the crossing points. Then, the transition (tunneling) from one diabatic surface to another occurs and it is followed by the subsequent relaxation of the reaction coordinate. If the tunneling coupling $\Delta$ is very small, then the tunneling presents a rate limiting step as the relaxation of the reaction coordinate is relatively fast. In this limiting case, no multiple crossings occur and the ET transfer is strictly nonadiabatic. To obtain the corresponding nonadiabatic ET rates we assume that initially the electron is either in the donor $(j=1)$ or acceptor $(j=2)$ state, with the reaction coordinate relaxed to its equilibrium probability density on the corresponding diabatic curve $V_{1}(x)$, or $V_{2}(x)$, i.e., $\rho_{j j}(x, 0)=P_{j}(0) g_{j}(x)$, where

$g_{j}(x)=\frac{\exp \left[-\frac{V_{j}(x)}{k_{\mathrm{B}} T}\right]}{\int_{-\infty}^{\infty} \mathrm{d} x \exp \left[-\frac{V_{j}(x)}{k_{\mathrm{B}} T}\right]}$.

Then, the full transfer rate and the equilibrium populations in Eq. (3) $\operatorname{read} \Gamma \approx \Gamma_{\mathrm{NA}}=k_{\mathrm{NA}}^{(1)}+k_{\mathrm{NA}}^{(2)}$ and $p_{j j}(\infty)=\left(\delta_{j, 1} k_{\mathrm{NA}}^{(2)}+\delta_{j, 2} k_{\mathrm{NA}}^{(1)}\right) /\left(k_{\mathrm{NA}}^{(1)}+k_{\mathrm{NA}}^{(2)}\right)$, where the forward $\left(k_{\mathrm{NA}}^{(1)}\right)$ and backward $\left(k_{\mathrm{NA}}^{(2)}\right)$ nonadiabatic rates are given by $[17,19,22]$

$k_{\mathrm{NA}}^{(j)}=\int_{-\infty}^{\infty} \mathrm{d} x K(x) g_{j}(x)$.

The full expression for $K(x)$ is rather involved and it will be presented elsewhere [22]. Inserting it into the above formula yields

$k_{\mathrm{NA}}^{(j)}=\frac{\Delta^{2} \Lambda_{j}^{1 / 2}}{\hbar^{2}} \int_{0}^{\infty} \mathrm{d} t \operatorname{Re}\left\{N_{j}(t) \exp \left[R_{j}(t)\right]\right\}$,

where

$$
\begin{aligned}
& N_{j}(t) \\
& =\alpha^{1 / 2} \exp \left[\frac{(1-\alpha) t}{2 \tau}\right] /\left\{(\alpha+1)\left[2 \Lambda_{j}+\frac{\alpha-1}{2}\right]\right. \\
& \left.\quad+(\alpha-1)\left[2 \Lambda_{j}-\frac{\alpha+1}{2}\right] \exp \left[-\frac{2 \alpha t}{\tau}\right]\right\}^{1 / 2},
\end{aligned}
$$

$$
\begin{aligned}
R_{j}(t)= & \mathrm{i}\left[\frac{\lambda_{1} \lambda_{2}\left(\Lambda_{1}-\Lambda_{2}+\mathrm{i} \chi\right)}{\hbar\left(\lambda_{1}+\lambda_{2}\right) \alpha^{2}}-\frac{\epsilon_{0}}{\hbar}\right] t \\
& +\frac{4 \tau \lambda_{1}^{2} \lambda_{2}^{2}}{\hbar \lambda_{j}\left(\lambda_{1}+\lambda_{2}\right)^{2} \alpha^{3}} \\
& \times\left\{\frac{\left[\mathrm{i}\left(2 \Lambda_{j}+1\right)(-1)^{j-1}+\chi\right] \alpha \sinh \left(\frac{\alpha t}{\tau}\right)}{4 \Lambda_{j} \alpha \cosh \left(\frac{\alpha t}{\tau}\right)+\left[4 \Lambda_{j}+\alpha^{2}-1\right] \sinh \left(\frac{\alpha t}{\tau}\right)}\right. \\
& \left.+\frac{2 \Lambda_{j}\left[2 \mathrm{i}(-1)^{j-1}+\chi\right]\left[\cosh \left(\frac{\alpha t}{\tau}\right)-1\right]}{4 \Lambda_{j} \alpha \cosh \left(\frac{\alpha t}{\tau}\right)+\left[4 \Lambda_{j}+\alpha^{2}-1\right] \sinh \left(\frac{\alpha t}{\tau}\right)}\right\},
\end{aligned}
$$


and we have defined the dimensionless parameters $\Lambda_{j}=\lambda_{j} /\left(\lambda_{1}+\lambda_{2}\right), \quad \chi=4 k_{\mathrm{B}} T \tau / \hbar, \quad$ and $\alpha=[1+$ $\left.\mathrm{i}\left(\Lambda_{1}-\Lambda_{2}\right) \chi\right]^{1 / 2}$. The remaining time integral in expression (8) can be calculated by a numerical quadrature. The above expression for the nonadiabatic rates is the first main result in this paper.

The formulas for the nonadiabatic rates simplify considerably if one makes use of the contact approximation, where $K(x)$ is given by

$K(x)=\frac{\pi \Delta^{2}}{2 \hbar} \delta\left[V_{1}(x)-V_{2}(x)\right]$.

This approximation is at the starting point of Tang's analysis of the Zusman equations. Within this contact approximation, the electronic transitions take place precisely at the crossing points.

Inserting Eqs. (6) and (11) for different parabolic surfaces into Eq. (7) yields for the forward and backward nonadiabatic rates, with $\epsilon_{0}<\epsilon_{\mathrm{c}}$

$$
\begin{aligned}
k_{\mathrm{NA}}^{(1)}= & \frac{\Delta^{2}}{4 \hbar} \sqrt{\frac{\pi}{k_{\mathrm{B}} T \lambda_{2}\left(1-\epsilon_{0} / \epsilon_{\mathrm{c}}\right)}} \\
& \times\left\{\exp \left[-\frac{\left(\lambda_{2}-\epsilon_{0}\right)^{2}}{4 \lambda_{+}\left(\epsilon_{0}\right) k_{\mathrm{B}} T}\right]\right. \\
& \left.+\exp \left[-\frac{\left(\lambda_{2}-\epsilon_{0}\right)^{2}}{4 \lambda_{-}\left(\epsilon_{0}\right) k_{\mathrm{B}} T}\right]\right\},
\end{aligned}
$$

$k_{\mathrm{NA}}^{(2)}=\sqrt{\frac{\lambda_{2}}{\lambda_{1}}} \exp \left(-\frac{\epsilon_{0}}{k_{\mathrm{B}} T}\right) k_{\mathrm{NA}}^{(1)}$,

where we have defined the auxiliary, bias-dependent quantities

$\lambda_{ \pm}\left(\epsilon_{0}\right)=\frac{\left[\lambda_{2} \pm \sqrt{\left(1-\epsilon_{0} / \epsilon_{\mathrm{c}}\right) \lambda_{1} \lambda_{2}}\right]^{2}}{4 \lambda_{1}}$.

The expressions in Eqs. (12) and (13) have also been obtained by Tang [14]. For equal curvatures the nonadiabatic rate expressions (12) and (13) reduce to the celebrated result by Marcus [1-3], Levich and Dogonadze [5].

The quality of the analytical rate expressions is illustrated in Fig. 2, where the full rate $\Gamma_{\mathrm{NA}}=$ $k_{\mathrm{NA}}^{(1)}+k_{\mathrm{NA}}^{(2)}$ is compared with the results obtained from a numerical integration of the Zusman equations in Eq. (1) for a range of energy bias $\epsilon_{0}$. We have used the standard NAG routine D03PCF
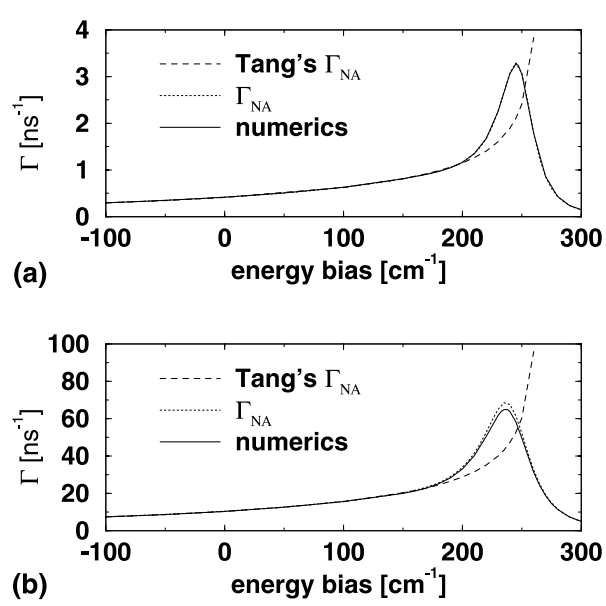

Fig. 2. Dependence of the full ET rate, $\Gamma$, on the energy bias, $\epsilon_{0}$. The parameters are: $\lambda_{1}=800 \mathrm{~cm}^{-1}, \lambda_{2}=200 \mathrm{~cm}^{-1}, T=$ $300 \mathrm{~K}, \Delta=1 \mathrm{~cm}^{-1}, \tau=1$ ps (a) and, $\Delta=5 \mathrm{~cm}^{-1}, \tau=0.5 \mathrm{ps}$ (b). The numerical results from Eq. (1) (solid line) are compared with those given by our nonadiabatic rate expression in Eq. (8) (dotted line), and Tang's expression in Eqs. (12) and (13) (dashed line).

for the following set of parameters: $\lambda_{1}=800 \mathrm{~cm}^{-1}$, $\lambda_{2}=200 \mathrm{~cm}^{-1}, T=300 \mathrm{~K}, \Delta=1 \mathrm{~cm}^{-1}, \tau=1 \mathrm{ps}$ (a) and, $\Delta=5 \mathrm{~cm}^{-1}, \tau=0.5 \mathrm{ps}$ (b) typical for ET in nonpolar solvents. Note however that we have chosen very different reorganization energies on purpose, in order to demonstrate the quality of the analytical results. In realistic situations, the relative difference between the reorganization energies should be much smaller $[9,10]$. The dashed lines correspond to Tang's expression, Eqs. (12), (13), the dotted lines to our new rate expressions, Eq. (8), and the solid lines to the numerical results. As shown in both panels in Fig. 2, the agreement of our analytical predictions and the numerical results is excellent for all the bias values considered. The slight deviation observed in the bottom panel is due to the fact that for this value of $\Delta$, the transition is not strictly nonadiabatic. On the other hand, the deviations of Tang's predictions from the numerical results become appreciable for $\epsilon_{0} \gtrsim 200 \mathrm{~cm}^{-1}$. This indicates a failure of the contact approximation. The two crossing points coalesce at $\epsilon_{0}=\epsilon_{\mathrm{c}} \approx 266 \mathrm{~cm}^{-1}$, where Tang's formula exhibits a divergence. For larger values of the bias $\epsilon_{0}$ the two diabatic surfaces are decoupled (no crossing point exists for $\epsilon_{0}>\epsilon_{\mathrm{c}}$ ) and the contact 
approximation makes no sense. We would also like to call the attention to the distinct asymmetric dependence of the full ET rate on the energy bias $\epsilon_{0}$ with respect to $\epsilon_{0} \rightarrow-\epsilon_{0}$. This feature is in a sharp contrast to the case of equal curvatures (not shown).

When the friction strength $\eta$ increases, the reaction coordinate slows down and at some point the simple nonadiabatic picture fails. Then, one needs to take into account multiple recrossings through the contact region, e.g., along the lines of $[17,19]$. This is achieved by solving iteratively Eq. (4), assuming that the initial conditions for the reaction coordinate are of the form given in Eq. (6). Detailed calculations will be presented elsewhere [22]. After using the sotermed 'consecutive step approximation' [17,19], which disentangles the effects of tunneling and diffusion, we obtain the following rate expression [22]:

$$
\begin{aligned}
k^{(1,2)}= & \frac{1+r_{1} r_{2} \sum_{j=1}^{2} \sum_{l=1}^{2} \sum_{n=1}^{2}(-1)^{l+n} \frac{k_{\mathrm{NA}}^{(j)}}{k_{D_{l n}}^{(j)}}}{\prod_{l=1}^{2}\left[1+r_{l} \sum_{j=1}^{2} \frac{k_{\mathrm{NA}}^{(j)}}{k_{D_{l l}}^{(j)}}\right]-r_{1} r_{2}\left[\sum_{j=1}^{2} \frac{k_{\mathrm{NA}}^{(j)}}{k_{D_{12}}^{(j)}}\right]^{2}} \\
& \times k_{\mathrm{NA}}^{(1,2)} .
\end{aligned}
$$

In Eq. (15), the coefficients $k_{D_{l n}}^{(j)}$ arise from the diffusional dynamics along the diabatic surfaces. They are given by

$$
\begin{aligned}
\frac{1}{k_{D_{l n}}^{(j)}}= & \tau_{j} \int_{0}^{\infty} \mathrm{d} s \\
& \times\left[\frac{\exp \left[\frac{\lambda_{j}}{2 k_{\mathrm{B}} T}\left(\frac{\left(y_{l}+y_{n}-2 \delta_{j, 2}\right)^{2}}{e^{s}+1}-\frac{\left(y_{l}-y_{n}\right)^{2}}{e^{s}-1}\right)\right]}{\left(1-e^{-2 s}\right)^{1 / 2}}-1\right] .
\end{aligned}
$$

The coefficients $r_{j}$,

$r_{j}=\frac{g_{1}\left(x_{j}^{*}\right)}{g_{1}\left(x_{1}^{*}\right)+g_{1}\left(x_{2}^{*}\right)}=\frac{g_{2}\left(x_{j}^{*}\right)}{g_{2}\left(x_{1}^{*}\right)+g_{2}\left(x_{2}^{*}\right)}$,

denote the weights of the two crossing points contributions. In Eq. (16) $y_{l}=x_{l}^{*} / x_{0}$ and $\tau_{j}$ are the relaxation time constants defined above. The full consecutive step rate is $\Gamma=k^{(1)}+k^{(2)}$. Eq. (15) represents a generalization of the consecutive step reaction rate first obtained by Zusman [6] to the case of two different curvatures: it bridges the ET dynamics between the nonadiabatic and the solvent controlled adiabatic ET regimes.

The method that we have used to derive Eq. (15) does not necessarily involve the contact approximation, Eq. (11). When such an approximation is invoked, the nonadiabatic rate constants in Eq. (15) are given by Eqs. (12) and (13). Tang [14] has previously derived a similar, but not identical, expression for the overall rate constant. His treatment differs from ours in two main aspects. First, in his derivation, the contact approximation is invoked from the very beginning. Second, the terms in Eq. (15) involving $k_{D_{12}}$ are neglected, as he assumes that the two crossing points are far apart. When one of the crossing points is much higher in energy than the other one, $r_{1} \simeq 1$ and $r_{2} \simeq 0$. In this case, expression (15) simplifies considerably to

$k^{(1,2)}=\frac{k_{\mathrm{NA}}^{(1,2)}}{1+k_{\mathrm{NA}}^{(1)} / k_{D_{11}}^{(1)}+k_{\mathrm{NA}}^{(2)} / k_{D_{11}}^{(2)}}$.

The coefficient $k_{D_{11}}^{(j)}$ can be expressed through the generalized hypergeometric series ${ }_{2} F_{2}(a, b ; c, d ; z)$ [23] as [19]

$$
\frac{1}{k_{D_{11}}^{(j)}}=\tau_{j}\left(\ln 2+2\left(\frac{E_{\mathrm{a}}^{(j)}}{k_{\mathrm{B}} T}\right){ }_{2} F_{2}\left(1,1 ; \frac{3}{2}, 2 ; \frac{E_{\mathrm{a}}^{(j)}}{k_{\mathrm{B}} T}\right)\right),
$$

where $E_{\mathrm{a}}^{(1)}=\left(\lambda_{2}-\epsilon_{0}\right)^{2} /\left(4 \lambda_{+}\left(\epsilon_{0}\right)\right)$ and $E_{\mathrm{a}}^{(2)}=E_{\mathrm{a}}^{(1)}+$ $\epsilon_{0}$ are the forward and backward activation energies from the diabatic potential bottoms to the first crossing point, respectively. It is also useful to note that for large activation energies, $E_{\mathrm{a}}^{(1,2)} \gg k_{\mathrm{B}} T$, this coefficient can be approximated as

$k_{D_{11}}^{(j)} \approx \tau_{j}^{-1} \sqrt{E_{\mathrm{a}}^{(j)} /\left(\pi k_{\mathrm{B}} T\right)} \exp \left(-E_{\mathrm{a}}^{(j)} / k_{\mathrm{B}} T\right)$

[19]. Note that the inverse of the consecutive step rate $k^{(1,2)}$ in (18) has the following structure $1 / k^{(1,2)}=1 / k_{\mathrm{NA}}^{(1,2)}+a_{1,2}$, where $a_{1,2} \propto \tau$ are the adiabatic corrections due to the multiple crossings through the reaction region. With increasing $\tau \sim \eta$ in (18) the nonadiabatic (tunneling step) contribution becomes negligible and a crossover to the 
solvent controlled adiabatic limit occurs. The corresponding adiabatic rates read

$$
\begin{aligned}
k^{(j)} \approx k_{\mathrm{ad}}^{(j)}= & \frac{1}{\eta} \frac{m \omega_{j}}{\sqrt{\pi k_{\mathrm{B}} T}} \frac{\omega_{1} \omega_{2} \sqrt{E_{\mathrm{a}}^{(1)} E_{\mathrm{a}}^{(2)}}}{\omega_{1} \sqrt{E_{\mathrm{a}}^{(1)}}+\omega_{2} \sqrt{E_{\mathrm{a}}^{(2)}}} \\
& \times \exp \left(-\frac{E_{\mathrm{a}}^{(j)}}{k_{\mathrm{B}} T}\right) .
\end{aligned}
$$

These rates cease to depend on the value of tunneling matrix element $\Delta$. For the case of equal curvatures, $\omega_{1}=\omega_{2}=\omega$, the adiabatic rate expression (20) reduces to the known result in $[6,19]$. Moreover, for equal curvatures and zero energy bias $\epsilon_{0}=0$ it reduces further to the Kramers rate expression for the cusp potential [24,25].

The comparison between analytical results and the numerical integration of Zusman Eq. (1) is depicted in Fig. 3 versus the relaxation time $\tau$ at fixed energy bias $\epsilon_{0}=0$. The other parameters are given in the caption of Fig. 3. With symbols we have plotted the numerical results; with solid line the prediction of Eq. (15) with $k_{\mathrm{NA}}^{(j)}$ given by Eq. (8); with dashed line the results obtained with the one crossing point approximation in Eq. (18) with $k_{\mathrm{NA}}^{(j)}$ given by Eq. (8); the dotted line represents the results obtained with Eq. (15) after neglecting the

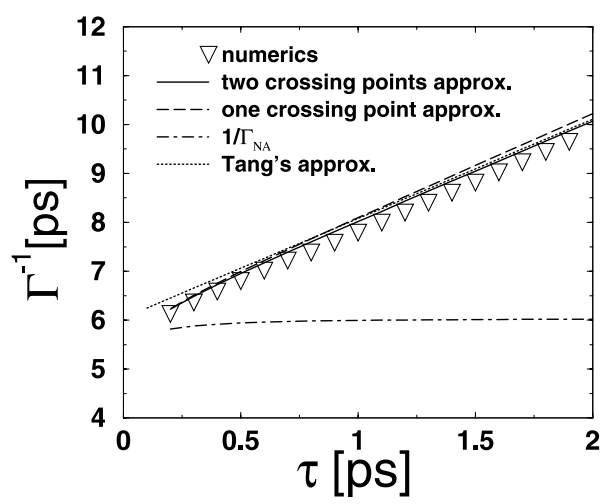

Fig. 3. Dependence of the inverse ET rate, $1 / \Gamma$, on the correlation time, $\tau$, for zero energy bias $\epsilon_{0}=0$. The other parameters are: $\lambda_{1}=800 \mathrm{~cm}^{-1}, \lambda_{2}=200 \mathrm{~cm}^{-1}, \Delta=20 \mathrm{~cm}^{-1}$, and $T=$ $300 \mathrm{~K}$. The depicted results are: the precise numerics in Eq. (1) (triangles); the two crossing points result in Eq. (15), (8) (solid line); the simplified result in (18), (8) (dashed line); the result obtained with Tang's approach (dotted line) and the nonadiabatic rate in Eq. (8) (dashed-dotted line).a terms containing $k_{D_{12}}^{(j)}$ and using $k_{\mathrm{NA}}^{(j)}$ in the contact approximation. This corresponds to the approximate result in [14]. Our nonadiabatic rate is also plotted with dashed-dotted line to show that the total rate approaches the nonadiabatic one as $\tau$ decreases. As $\tau$ increases, the inverse rate is proportional to the relaxation time, $1 / \Gamma \propto \tau$, in agreement with the analytical results. This clearly indicates the transition to the solvent controlled adiabatic regime with increasing friction. Note that for $\epsilon_{0}=0$ all the analytical expressions for $k_{\text {NA }}^{(j)}$ yield good results (cf. Fig. 2), so that, both Tang's and our expressions provide good agreement with the numerics. This is not necessarily the case when the energy bias gets larger. Then, an adequate evaluation of the nonadiabatic rates is important.

Moreover, Fig. 3 demonstrates that the single crossing point approximation in Eqs. (18) and (19) can be proposed for convenient experimental use, in view of its relative simplicity. For a smaller difference between the curvatures the agreement is improved further (not shown).

In conclusion, we have presented an extension of Zusman approach to ET problems to the case of diabatic potentials with different curvatures. We obtain new analytical expressions for the nonadiabatic and solvent controlled adiabatic rates. The validity of our analysis has been tested by comparison with the numerical solution of Zusman equations in a wide range of parameters. The authors are confident in believing that Eqs. (8)-(10) and, in particular, (12)-(14) and (18), (19) will prove useful for future theoretical work and, more importantly, for the analysis of experimental results of ET-works in condensed phases.

\section{Acknowledgements}

The authors gratefully acknowledge support of this work by the Deutsche Forschungsgemeinschaft within the Acciones Integradas (Grant 314/ Al-e-dr), within the SFB 486, project A10 (I.G. and P.H.), and by Grant A/01/19459 from DAAD (J. Casado-Pascual). We thank also C. Denk, J. Lehmann, and A. Nitzan for useful discussions. 


\section{References}

[1] R.A. Marcus, J. Chem. Phys. 24 (1956) 966.

[2] R.A. Marcus, J. Chem. Phys. 26 (1957) 867.

[3] R.A. Marcus, Rev. Mod. Phys. 65 (1993) 599.

[4] N.S. Hush, J. Chem. Phys. 28 (1958) 962.

[5] V.G. Levich, R.R. Dogonadze, Dokl. Akad. Nauk SSSR 124 (1959) 123.

[6] L.D. Zusman, Chem. Phys. 49 (1980) 295.

[7] I.V. Alexandrov, Chem. Phys. 51 (1980) 449.

[8] T. Kakitani, N. Mataga, J. Phys. Chem. 89 (1985) 4752.

[9] W.W. Parson, Z.T. Chu, A. Warshel, Biophys.J. 74 (1998) 182.

[10] C. Denk, M. Morillo, F. Sánchez-Burgos, A. Sánchez, J. Chem. Phys. 110 (1999) 473.

[11] P. Pérez-Tejada, F.J. Franco, A. Sánchez, M. Morillo, C. Denk, F. Sánchez-Burgos, PCCP 3 (2001) 1271.

[12] C. Hartnig, M.T.M. Koper, J. Chem. Phys. 115 (2001) 8540.

[13] H.-X. Zhou, A. Szabo, J. Chem. Phys. 103 (1995) 3481.

[14] J. Tang, Chem. Phys. 188 (1994) 143.
[15] J. Jortner, M. Bixon (Eds.), Adv. Chem. Phys. 106 \& 107 (Parts I \& II) (1999).

[16] A. Garg, J.N. Onuchic, V. Ambegaokar, J. Chem. Phys. 83 (1985) 4491.

[17] D.Y. Yang, R.I. Cukier, J. Chem. Phys. 91 (1989) 281.

[18] P.A. Frantsuzov, J. Chem. Phys. 111 (1999) 2075.

[19] L. Hartmann, I. Goychuk, P. Hänggi, J. Chem. Phys. 113 (2000) 11159;

L. Hartmann, I. Goychuk, P. Hänggi, J. Chem. Phys. 115 (2001) 3969 (erratum).

[20] I. Goychuk, L. Hartmann, P. Hänggi, Chem. Phys. 268 (2001) 151.

[21] M. Thoss, H. Wang, W.H. Miller, J. Chem. Phys. 115 (2001) 2991

[22] J. Casado-Pascual, I. Goychuk, M. Morillo, P. Hänggi (in preparation).

[23] I.S. Gradstein, I.M. Ryzhik, Table of Integrals, Series, and Products, Academic Press, New York, 1965.

[24] H.A. Kramers, Physica (Utrecht) 7 (1940) 284.

[25] P. Hänggi, P. Talkner, M. Borkovec, Rev. Mod. Phys. 62 (1990) 251. 